\title{
Agôn
}

Revue des arts de la scène

$\varnothing \mid 2007$

En quête du sujet

\section{Le sujet hors-scène}

Lecture de trois pièces de Jean-Luc Lagarce

\section{Aurélie Coulon}

\section{(2) OpenEdition}

Journals

Édition électronique

URL : http://journals.openedition.org/agon/305

DOI : 10.4000/agon.305

ISSN : 1961-8581

Éditeur

Association Agôn

Référence électronique

Aurélie Coulon, "Le sujet hors-scène », Agôn [En ligne], $\varnothing \mid$ 2007, mis en ligne le 13 décembre 2007, consulté le 17 avril 2020. URL : http://journals.openedition.org/agon/305 ; DOI : https://doi.org/ 10.4000/agon.305

Ce document a été généré automatiquement le 17 avril 2020.

Association Agôn et les auteurs des articles 


\title{
Le sujet hors-scène
}

\author{
Lecture de trois pièces de Jean-Luc Lagarce
}

\author{
Aurélie Coulon
}

1 Le sujet lagarcien est d'abord sujet de parole : c'est en parlant qu'il se crée, c'est ainsi que surgit le personnage mais aussi l'espace de jeu qui progressivement se dessine. On ne sait si ceux qui se trouvent en scène sont des personnages, des acteurs ou des êtres de fiction venus raconter - rejouer ? - un drame déjà vécu car cette dramaturgie crée des êtres pour la scène dont la spécificité réside précisément dans cette ambiguïté ${ }^{1}$. Mais au-delà du statut problématique des personnages-acteurs, le théâtre de J-L. Lagarce brouille à plusieurs niveaux les acceptions de la notion de sujet. Absent comme le jeune homme de J'étais dans ma maison..., le sujet peut demeurer hors-scène et être " raconté " par les personnages présents sur le plateau. Il est alors presque objectivé ; c'est un sujet au sens premier du terme : ce dont on parle. Comme il est opaque, les autres cherchent à faire de lui un objet de discours. Second sens de la notion, et second niveau d'incertitude : on appelle sujet une personne, un individu. De ce point de vue aussi, les catégories sont brouillées: sujet fragmenté, sujet tourné vers son passé, sujet dont l'individualité se dilue dans le flux d'une langue commune à tous les personnages, sujet dont la présence « ici et maintenant $\rrbracket^{2}$ peut toujours être mise en doute. Si on considère qu'un sujet se caractérise par son aptitude à mettre en oeuvre une parole de manière individuelle, le statut des personnages lagarciens devient incertain : ces êtres parlent tous la même langue, qu'on peut définir succinctement en mentionnant la longueur de la phrase, ses volutes, son déploiement en un perpétuel mouvement d'autocorrection, la tendance à l'énonciation citationnelle, le souci du mot juste. Il y a nivellement, voire anéantissement de la singularité par l'usage de cette langue commune; cependant des individus se dessinent autrement, identités problématiques. Dans Juste la fin du monde et Le Pays lointain cette parole désindividualisée circule d'un personnage à l'autre et devient vacillante dès lors qu'il s'agit de reconstituer le parcours et l'identité de Louis et de faire ainsi l'archéologie du sujet; la mémoire même est fiction. Dans certains cas, le personnage ne peut être assimilé à un individu, il y a alors diffraction du sujet au sein d'un choeur ou d'une entité collective. Tenter de définir le sujet lagarcien, c'est se confronter à ses incomplétudes : fragmentaire, voire pluriel, étranger aux autres et à soi-même, travaillé par un passé souvent mal connu, il semble toujours relégué ailleurs. 
Dans Juste la fin du monde, J'étais dans ma maison et j'attendais que la pluie vienne et Le Pays lointain, le sujet se définit ainsi entre présence et absence. Parfois absent et relégué hors-scène, il peut aussi avoir une présence problématique, lorsqu'il est incarnation d'un mort de retour parmi les vivants ou lorsqu'il oscille entre les fonctions de personnage, d'acteur, ou de narrateur. D'autre part, le passé des personnages reste bien souvent inconnaissable. Ces identités troubles ne sont dévoilées que partiellement: difficulté à dire, insuffisances de la mémoire les vouent à l'incertitude. Dans ces trois pièces, le drame est déjà joué depuis longtemps : un jeune homme a quitté sa famille à la suite d'une dispute avec son père. La teneur exacte de cette dispute échappe au spectateur et aux personnages. La catastrophe est lointaine, cette scène a été oubliée, déformée à force d'être ressassée. Incertitude concernant le passé, concernant l'identité et l'aptitude du langage à dire quelque chose de l'énonciateur, portant sur la nature même des personnages-acteurs qui sont en scène : le sujet lagarcien ne semble pouvoir s'appréhender que par le biais de ce qui, en lui, nous échappe. Pour esquisser un sujet lagarcien, peut-être faut-il d'abord repérer ses points de fuite, ses manques, ses zones d'ombres ; ici, le hors-scène.

2 Par hors-scène, j'entends tout ce qui est hors du champ de vision du spectateur (espaces contigus au plateau et possibles prolongements du lieu scénique), les scènes non représentées et les personnages absents évoqués au cours des narrations. Le horsscène est généralement associé au champ sémantique de l'espace; dans le cas de la dramaturgie lagarcienne la notion semble cependant se définir plus par la temporalité qu'elle désigne que par les données spatiales, souvent vagues. Souvent, le passé hante des personnages qui ne cessent de le raconter en variant les versions et les formulations : c'est un théâtre du dire dont tout l'enjeu se situe sur le terrain de la parole, de la mise en récit du passé, et c'est précisément de cette matière narrative que naissent les situations dramatiques. C'est à partir de ce flux narratif que se constitue le hors-scène. Le texte théâtral, toujours pensé en vue d'un devenir scénique, a sa propre spatialité et sa propre manière de produire des images; j'ai cherché à comprendre quelles pouvaient être les conditions de l'avènement des images qui ne sont pas montrées, mais qui sont cependant vues par le spectateur et situées dans un autre lieu nommé hors-scène. Je les qualifierai de visuelles, empruntant cette notion à G. DidiHuberman qui l'applique notamment au blanc "présent» d'une fresque de Fra Angelico, fresque qui fait l'objet d'une véritable leçon de regard en ouverture de Devant l'image. Paradoxalement, cette Annonciation se trouve sur un mur percé d'une fenêtre et le contre-jour empêche d'abord le spectateur de bien la voir. Elle se détache mal du blanc du mur, de ce blanc qui lui sert de fond. C'est précisément la puissance de ce blanc qui retient l'attention de G. Didi-Huberman, et l'auteur forge des outils d'analyse pour ce phénomène qui, comme le hors-scène, échappe au moins en partie à l'observation :

Tel serait donc le nouveau terme à introduire, à distinguer du visible (en tant qu'élément de représentation, au sens classique du mot) comme de l'invisible (en tant qu'élément d'abstraction). Le blanc d'Angelico fait évidemment partie de l'économie mimétique de sa fresque [...]. A ce titre, il appartient bien au monde de la représentation. Mais il l'intensifie hors de ses limites, il déploie autre chose, il atteint son spectateur par d'autres voies. ${ }^{3}$

3 Il en est du hors-scène comme du blanc de la fresque de Fra Angelico : « Il n'est pas rien, puisqu'il nous atteint sans que nous le puissions saisir, et qu'il nous enveloppe sans que nous le puissions, à notre tour, prendre dans les rets d'une définition. ${ }^{4}{ }^{\prime} C^{\prime}$ est 
un objet d'étude qui ne peut être abordé que de manière indirecte, par le biais des éléments du texte dramatique qui servent de jalons pour l'imaginaire ou encore du point de vue des effets qu'il peut produire sur le spectateur. Le hors-scène sera envisagé comme processus, comme devenir image ou devenir représentation, comme manifestation d'une théâtralité qui reste à définir. Si dans la dramaturgie lagarcienne le sujet se définit entre scène et hors-scène, cela semble créer un type particulier de présence théâtrale et non spectaculaire, présence toujours à construire par le spectateur, présence résolument recentrée autour de la figure de l'acteur et de la temporalité de la représentation. A travers l'étude de Juste la fin du monde, de J'étais dans ma maison et j'attendais que la pluie vienne et du Pays lointain je tenterai de dégager la spécificité de la présence hors-scène du sujet. Sujet qui persiste aux marges de la représentation, comme un souvenir des anciennes formes du personnage dramatique ou comme l'ombre des personnages-acteurs, entre deux espaces et entre deux temporalités. "[C]omme une esquisse de ce que cela aurait pu être ou de ce que cela fut $»^{5}$

\section{Le sujet à construire : indétermination du passé, identités fragmentaires}

\section{Difficultés d'énonciation et soupçon sur le langage}

Les personnages lagarciens ne sont donnés à percevoir au spectateur que par morceaux. Ces fragments d'identité sont contradictoires : ces êtres se méconnaissent et peinent à raconter leur histoire, souvent déclinée en différentes versions. C'est que le langage même apparaît comme un medium contestable. Le monologue de Louis, prologue de Juste la fin du monde, n'a pas seulement une fonction informative. Louis explique qu'il revient dans sa famille après plusieurs années d'absence pour annoncer sa mort " prochaine et irrémédiable ${ }^{6} »$. Juste la fin du monde c'est l'histoire de ce retour, des retrouvailles complexes avec une famille devenue lointaine et de l'impossibilité de Louis à atteindre l'objectif qu'il s'était fixé. Détours, hésitations, épanorthoses : la manière de parler du personnage dit déjà quelque chose de son rapport au monde. C'est cette langue si particulière à J-L. Lagarce, qui semble d'abord quotidienne, voire familière à l'oreille mais qui s'avère travaillée de l'intérieur par des motifs thématiques et syntaxiques récurrents. Les personnages lagarciens font un usage distancié de la langue, ils ne cessent de commenter et de corriger leur discours. Ils cherchent le mot juste. Si le personnage selon Robert Abirached se situe quelque part « entre le mot et le corps ${ }^{7}$, le personnage chez J-L. Lagarce semble naître d'abord du mot. Selon Julie Sermon, le premier critère de définition des personnages lagarciens est en effet de nature linguistique. Ils s'identifient à leur manière de parler, qui fait d'eux une "communauté linguistique». Ces personnages hésitants à la parole hantée d'incertitudes sont des «créatures de langue [...] porteuses d'une même matière obsessionnelle et d'une même rythmique d'énonciation. ${ }^{8}$ "

5 Louis fait partie de cette communauté. Ainsi, son monologue initial dans Juste la fin $d u$ monde pose un personnage qui peine à formuler, à énoncer. C'est une parole répétitive et elliptique. Si on réduisait ce monologue à l'essentiel d'un point de vue informatif, il resterait une seule phrase : «Plus tard, l'année d'après, je décidai de retourner les voir, revenir sur mes pas et faire le voyage pour annoncer ma mort prochaine et irrémédiable, en être l'unique messager, me donner et donner aux autres une dernière 
fois l'illusion d'être responsable de moi-même.» Mais ce monologue se caractérise par de multiples hésitations, des remarques métalinguistiques, des ruptures temporelles soulignées par les ruptures typographiques.

Plus tard, l'année d'après - j'allais mourir à mon tour -

j'ai près de trente-quatre ans maintenant et c'est à cet âge que je mourrai,

l'année d'après,

de nombreux mois déjà que j'attendais à ne rien faire, à tricher, à ne plus savoir,

de nombreux mois que j'attendais d'en avoir fini,

l'année d'après,

comme on ose bouger parfois,

à peine,

devant un danger extrême, imperceptiblement, sans vouloir faire de bruit ou

commettre un geste trop violent qui réveillerait l'ennemi et vous détruirait

aussitôt,

l'année d'après,

je décidai de retourner les voir, revenir sur mes pas, aller sur mes traces et faire le

voyage,

pour annoncer, lentement, avec soin, avec soin et précision

- ce que je crois -

lentement, calmement, d'une manière posée

- et n'ai-je pas toujours été pour les autres et eux, tout précisément, n'ai-je pas

toujours été un homme posé ?,

pour annoncer,

dire,

seulement dire,

ma mort prochaine et irrémédiable, ${ }^{9}$

6 Ce monologue est un exemple particulièrement significatif de la langue parlée par les personnages lagarciens. Langue, et non parole : la parole suppose l'existence d'un sujet parlant, c'est une mise en oeuvre individuelle par laquelle l'appropriation du code linguistique se réalise dans un discours. La langue est un système de signes fonctionnant comme un code, reposant sur des conventions, et préexistant aux membres de la communauté qui l'utilisent à des fins de communication. D'un point de vue linguistique la singularité des personnages lagarciens peut être mise en doute : les dialogues semblent souvent mettre en circulation un flux unique qui passerait d'un personnage à l'autre sur le mode de l'enchaînement choral. Louis se distingue cependant des membres de sa famille de par sa propension à l'autocorrection, mais surtout en raison de son goût pour le récit. Il est le seul à savoir écrire, à maîtriser la fiction. C'est une figure du ressassement qui se raconte et se rejoue les histoires du passé. Son frère, Antoine, se méfie des histoires et n'a pas la même aptitude au récit. C'est peut-être la cause de la rivalité qui oppose les deux frères depuis l'enfance.

C'est cela,

c'est exactement cela, ce que je disais,

les histoires,

et après on se noie

et moi,

il faut que j'écoute et je ne saurai jamais ce qui est vrai

et ce qui est faux,

la part du mensonge.

Tu es comme ça,

s'il y a bien une chose

(non, ce n'est pas la seule!),

s'il y a bien une chose que je n'ai pas oubliée en songeant à toi, 
c'est tout cela, ces histoires pour rien,

des histoires, je ne comprends rien. ${ }^{10}$

7 Pourquoi Louis ne parvient-il pas à annoncer sa mort à sa famille, malgré ses facilités de narrateur? Juste la fin du monde, est-ce autre chose que le drame d'un homme qui ne parvient pas à annoncer - énoncer - sa mort, ni à raconter son histoire? C'est un drame de l'énonciation. L'histoire banale de l'échec de la fonction référentielle du langage : Louis ne réussit pas à transmettre son message, comme s'il lui était impossible d'énoncer sa propre disparition. Une part de son identité restera méconnue, condamnée à l'indétermination. Il écrit, certes, mais il ne sait pas dire. Un seul personnage sait énoncer : c'est Catherine, la femme d'Antoine, maladroite et effacée en apparence. Suzanne ne s'y trompe pas et la définit ainsi :

Suzanne. - Cette fille-là, on ne croit pas, la première fois où on la regarde, on la suppose fragile et démunie, tuberculeuse ou orpheline depuis cinq générations,

mais on se trompe,

il ne faut pas s'y fier :

elle sait choisir et décider,

elle est simple, claire, précise,

Elle énonce bien. ${ }^{11}$

8 D'un point de vue linguistique Suzanne est donc un sujet mieux défini, plus affirmé que Louis. Cette difficulté à dire ce qui doit être dit qui affecte les personnages lagarciens entraîne l'impossibilité de fournir une définition de soi stable. Pour le spectateur, les quelques informations sur le passé de Louis restent soumises au doute et à l'incertitude d'un hors-scène qui désigne ici une époque révolue, à peine esquissée à travers les silences et les failles des tentatives de récit dont elle est l'objet.

\section{La mémoire comme fiction de soi}

9 Le personnage de Louis, qui apparaît dans Juste la fin du monde et dans Le Pays lointain, est un être fragmentaire qui ne cesse de parler d'un passé qu'il connaît mal. Car l'enjeu des dialogues avec sa famille concerne la manière dont son histoire doit être racontée. Il est difficile d'accéder à un récit cohérent de son passé : les troubles de la mémoire et les insuffisances du langage employé par les personnages s'associent pour miner les tentatives de narration de zones d'ombre et d'éléments hypothétiques, voire inconnaissables. Eléments relégués hors-scène, dans la mesure où ils concernent directement le personnage en scène, auquel on suppose une existence au-delà des quelques épisodes représentés sur le plateau. André Bazin analyse ainsi ce phénomène : lorsqu'un personnage sort de scène "nous admettons qu'il échappe au champ visuel, mais il continue d'exister identique à lui-même en un autre point du décor, qui nous est caché ${ }^{12}$. Dans Juste la fin du monde (et dans Le Pays lointain, qui en est une réécriture) la parole de Louis est souvent confisquée par des proches qui croient le connaître mieux que lui-même : ces récits fragmentaires et fictifs en font une identité complexe dont certaines zones troubles demeurent hors-scène. Le sujet - Louis - est dépossédé d'une parole qui aurait pu faire de lui un individu à part entière. D'autres parlent comme lui, certains parlent à sa place. Du fait de ses longues années d'absence, ses proches se sont construit une image de lui et cette image persiste malgré son retour. La Mère de Louis raconte comment elle imagine les retrouvailles de Louis avec son frère et sa soeur et annonce comment il se comportera sans lui laisser le moindre espace de parole.

Et tu ne comprendras pas, je sais comment cela se passera et s'est toujours passé.

Tu répondras à peine deux ou trois mots 
et tu resteras calme comme tu appris à l'être par toi-même

- ce n'est pas moi ou ton père,

ton père encore moins,

ce n'est pas nous qui t'avons appris cette façon si habile et détestable d'être paisible

en toutes circonstances, je ne m'en souviens pas

ou je ne suis pas responsable -

tu répondras à peine deux ou trois mots,

ou tu souriras, la même chose,

tu leur souriras ${ }^{13}$

10 Dans Le Pays lointain le traitement du personnage de Louis change. Le point de vue est recentré sur le drame intime, mais Louis n'est plus un personnage sans passé comme dans Juste la fin du monde. Il est entouré de sa famille inventée, de ceux qu'il a croisés tout au long de sa vie: le passé est ainsi comme incarné. Il ne vient pas seul, et les membres de la famille qu'il s'est créée prennent parfois le relais et font à plusieurs voix le récit - toujours incomplet, toujours hypothétique - de sa vie. Car dans cette dramaturgie la mémoire est fictive : c'est toujours une réinvention de soi.

11 Certains des monologues prononcés par Louis dans Juste la fin du monde sont repris tels quels dans Le Pays lointain. Mais ils sont parfois pris en charge soit par «l'Amant, mort déjà ", soit par Longue Date. Ils sont transformés en conversations réunissant Louis et ces deux personnages. C'est ainsi que le Prologue est recomposé et devient un dialogue.

Louis. - Plus tard, l'année d'après.

L'Amant, mort déjà. - Une année après que je meurs, que je suis mort?

Louis. - Exactement ça. L'année d'après,

j'étais resté là, seul, abandonné, toutes ces sortes de choses,

plus tard, l'année d'après,

- j'allais mourir à mon tour -

(j'ai près de quarante ans maintenant et c'est à cet âge que je mourrai)

l'année d'après, je décidai de revenir ici. Faire le chemin à l'inverse.

Longue Date. - Histoire d'un jeune homme qui décide de revenir sur ses traces, revoir sa famille, son monde, à l'heure de mourir.

Histoire de ce voyage et de ceux-là, perdus de vue, qu'il rencontre et retrouve. ${ }^{14}$

12 Cette redistribution du texte amène une interrogation concernant le statut de ces deux personnages: L'Amant et Longue Date pourraient être des entités nées d'une subjectivité, issues des monologues intérieurs de Louis. Ils l'accompagnent partout, ne serait-ce qu'en souvenir, dans le cas de l'Amant. Il y a un rapport d'identité complexe entre ces trois personnages. Complexe, car ce ne sont pas des doubles de Louis : ils lui ressemblent seulement. L'Amant est mort, et Louis doit mourir un an plus tard. L'un des monologues de Louis dans Juste la fin du monde est désormais pris en charge par l'Amant :

L'Amant, mort déjà. - Au début, ce que l'on croit

- j'ai cru cela -

ce qu'on croit toujours, je l'imagine, c'est rassurant, on a moins peur, on se répète à soi-même cette solution comme aux enfants qu'on endort, ce qu'on croit un instant,

on l'espère, c'est que le reste du Monde, lorsqu'on mourra, c'est que le reste du Monde disparaîtra avec soi,

que le reste du Monde pourrait disparaître avec soi, s'éteindre, s'engloutir et ne plus me survivre. ${ }^{15}$ 

en a l'expérience. Cependant ces faits de réécriture ne suffisent pas à affirmer la nature intrasubjective des personnages de L'Amant et de Longue Date. Ce sont plutôt des narrateurs qui connaissent son histoire et qui semblent convoqués là pour la raconter. Ce sont moins des doubles que des voix qui traduisent la complexité d'une mémoire qui échappe parfois au sujet, d'une mémoire dialogique. Cela permet la spatialisation d'un sujet qui n'a pas nécessairement la maîtrise de tous ses souvenirs et qui a parfois besoin d'être secondé par d'autres voix. Représentation non mimétique d'une mémoire à la fois individuelle et éclatée qui rend compte d'une "coupure entre le moi et le réel ", qui est selon R. Abirached l'une des manifestations de l'influence de la psychanalyse sur la théorie de l'acteur :

L'expérience capitale, qu'il importe de traduire à la scène, serait alors celle d'une coupure entre le moi et le réel, corporellement et spirituellement ressentie : au personnage, par sa parole, et par son action dans l'espace, il reviendrait de cristalliser ce sentiment diffus de séparation et d'étrangeté. ${ }^{16}$

Des personnages tels que le Garçon ou le Guerrier ne sont-ils pas représentatifs d'une telle expérience? Ce sont des figures nées du moi qui personnifient les souvenirs de Louis en venant jouer le rôle de tous les « personnages de sa vie » :

Louis. - Les personnages, tous les personnages, ceux qu'on rencontre, qu'on voit, qui interviennent, les personnages évoqués, leur voix, juste leur photographie, toute la multitude des gens rencontrés, croisés, une nuit, une heure, dix minutes, juste un regard, on ne saurait les retenir, ou dans l'embrasure d'une porte, on passait à pied, trop tard, on voit l'Autre dans l'embrasure d'une porte et jamais on ne retrouvera l'endroit exact, ceux-là encore avec qui on partagea tout, presque tout - on se le promettait - dix ou vingt années entières, ceux avec qui on « fit »sa vie et au-delà encore.

L'Amant, mort déjà. - Moi.

Louis. - Toi.

Et la foule encore des autres, essentiels, à peine entraperçus, ceux-là qui sont tous les autres personnages de notre vie. Tous ceux-là qui font la vie d'un seul homme. ${ }^{17}$

15 L'identité de Louis a été nourrie par tous les personnages qu'il a croisés durant sa vie. Le Garçon et le Guerrier sont des " personnages de l'individu », pour reprendre le titre de la pièce de Saint-Pol-Roux qui met en scène des êtres intrasubjectifs composant, une fois réunis sur le plateau, un individu ${ }^{18}$. Ils viennent faire leur énumération, suggérant sans les incarner - quels pouvaient être ces personnages parfois à peine croisés par Louis. C'est une galerie de portraits, voire d'esquisses :

Un Garçon, tous les garçons. - Celui-là qui devient fou, on le connaissait enfant, on le quitta, on le retrouve, doucement, ce que dit ta mère, il devient fou.

Je ne dis pas dans l'ordre.

Celui-là qu'on voit parfois, qu'on croise, claveciniste et militaire à Châteauroux, j'en ai déjà parlé. Il habite vers Denfert, en te débrouillant bien, à certaines heures certains après-midi, tu peux le rencontrer par le plus grand des hasards chez l'Arabe qui fait le coin, en descendant la rue du Faubourg-Saint-Jacques. Les premiers temps, tu ne fais plus tes courses que là, c'est loin de ta maison, les fruits et légumes ne sont pas très bons, le résultat est aléatoire. ${ }^{19}$

Le Garçon et le Guerrier sont des personnages en puissance, figures neutres qui sont là pour représenter les absents et porter leur empreinte. Parmi les personnages du drame, il y en a donc certains qui seront suggérés sans être véritablement incarnés. Le "garçon 
qui devient fou » restera une figure du hors-scène. Les entités collectives du théâtre de J-L. Lagarce ne sont pas des personnages au sens traditionnel du terme mais plutôt des porteurs de mémoire qui dialoguent avec Louis :

Un Garçon, tous les garçons. - Thomas, à Berlin encore, s'étant rhabillé et descendu avec toi pour attendre à tes côtés un taxi sous la pluie. Pour mention. (J'aurai besoin d'un parapluie pour unique accessoire, la pluie nous la devinerons.)

Nous serons ainsi, Thomas et toi, à ne plus rien savoir nous dire, on ne se reverra plus, nous le savons et on ne se regrettera même pas, et nous savons aussi que nous ne nous regretterons pas, et c'est toujours à cette aune-là, l'aune du regret que nous jugeons de l'importance de la scène, nous serons ainsi, côte à côte, ensemble, oui, ensemble, comme peu le furent, sous la pluie, pour quelques minutes d'éternité.

Louis. - Comment est-ce que tu as dit qu'il s'appelait ? ${ }^{20}$

En ce sens aussi le moi de Louis est collectif : le Garçon peut lui rappeler des souvenirs dont il a perdu la mémoire. Tout "tourne" autour de lui mais son point de vue ne devient jamais dominant, car les autres personnages l'aident à raconter son histoire et viennent réactiver les événements passés : ce sont des relais entre le passé hors-scène de Louis et la temporalité de la représentation. Entre deux espaces. Entre présence et absence. Si la mémoire se fait fiction, c'est à la fois parce qu'aucun des personnages ne connaît la véritable version des faits et parce que, dans Le Pays lointain, Le Garçon et Le Guerrier semblent parfois répéter en vue d'une future "mise en scène" des personnages de la vie de Louis. A l'horizon de l'évocation des souvenirs, une fiction théâtrale à venir se dessine. Dans cette dramaturgie la mémoire s'élabore entre fiction de soi et élan vers des moments oubliés dont il s'agirait de retrouver la substance. Le sujet lagarcien est tourné vers un passé qui lui échappe sans cesse : de ce point de vue, ce passé exclu de l'espace de représentation est l'objet d'un regard impossible sur les scènes oubliées d'une vie. Ce phénomène n'est pas sans évoquer une manifestation du hors-scène repérée par Arnaud Rykner notamment dans le théâtre de Marguerite Duras :

L'ambiguïté et l'extrême force de l'oeuvre de Marguerite Duras, par exemple, tient justement à sa capacité d'entretenir dans le texte la nostalgie d'un regard à la fois impossible et nécessaire, d'un au-delà des mots dont les yeux sont le seuil. ${ }^{21}$

Nostalgie d'un regard : de même, le sujet lagarcien est tourné vers un passé qui ne cesse de lui échapper. Il se constitue par rapport à cette ligne de fuite. Ce mouvement vers une histoire oubliée trouve sa pleine réalisation dans J'étais dans ma maison... : le jeune homme n'entre pas en scène, il ne se raconte pas, il est raconté. Il est sujet au sens premier de la notion : sujet de conversation.

\title{
J'étais dans ma maison et j'attendais que la pluie vienne : la disparition de Louis?
}

\author{
La Mère. \\ Il faut l'attendre, écouter les bruits, tendre l'oreille et chercher à peine, ne serait-ce \\ qu'à peine, \\ et chercher, à peine, de son lit, à guetter le souffle, \\ et voler les indices, les indices infimes qui nous le ramèneraient à la vie, \\ l'instant précis, \\ le même moment exact du temps où il était enfant, où il s'éveillait et commençait à \\ diriger aussitôt la maison, le jeune fils, à la faire tourner autour de lui, car toujours \\ elle tourna autour de lui ${ }^{22}$
}


Une maison qui «tourne » autour d'un frère absent, une famille qui ne parvient pas à voir autre chose que l'image fantasmée de celui-ci, construite durant de longues années, alors qu'il est désormais de retour... L'intrigue de J'étais dans ma maison... est très proche de celle de Juste la fin du monde. Le fils absent n'a plus de prénom, c'est désormais «Le Jeune Frère ». Il restera hors-scène, ce qui lui confère une présence accrue. Car tout tourne autour de lui.

Le Jeune Frère, c'est Louis du point de vue de sa famille. Elles ne savent plus rien de lui depuis des années, alors elles racontent les histoires de l'époque où il était encore là, elles imaginent ce qu'il a pu devenir. A partir des conversations et des récits des cinq femmes, l'imagination du spectateur est sans cesse sollicitée et chacun se crée une représentation du Jeune Frère d'après les jalons présents dans le texte. On veille un mort qui est tout près, dans la pièce du dessus, et ce lieu peut être désigné de temps à autre d'un regard ou d'un signe de tête. Ainsi se crée un lieu imaginaire adjacent à l'espace scénique. A cette présence latente s'ajoute une présence du jeune homme dans les conversations entrecoupées de récits des cinq femmes. Il est sans cesse évoqué, désigné par des périphrases telles que «le Jeune Frère " ou «le jeune fils", par des pronoms personnels ou démonstratifs comme «celui-là ». L'absence de prénom et d'incarnation scénique entraînent un effet de "désindividualisation ». Ainsi s'élabore un sujet pluriel dont la présence diffuse n'est ni celle de l'acteur ni celle du personnage.

21 L'espace scénique est surplombé par la chambre du jeune homme, chambre où les cinq femmes l'ont porté, inerte. Il y repose déjà lorsque la pièce commence. Aucune didascalie, mais une séquence entière est consacrée à cette chambre : dans le théâtre de J-L. Lagarce c'est la parole du personnage-acteur qui crée son espace de jeu. C'est au fil des dialogues que s'affirment l'existence et la fonction de la chambre. Cette pièce est une sorte de sanctuaire, rien n'y a été changé depuis le départ du Jeune Frère. A plusieurs reprises, les femmes répètent: "il est dans sa chambre » comme pour se persuader qu'il est bien présent. La Mère évoque ainsi ce lieu situé quelque part «audessus » de la scène :

La Mère. - [..] et pour les filles, celles-là, tu peux les voir depuis qu'il est revenu, depuis qu'il est couché et qu'il dort, dans sa chambre, là-haut, tu peux les voir, pour les filles non plus, celles-là, ce n'est pas rien. ${ }^{23}$

Cinq femmes parlent au rez-de-chaussée de leur maison, mais il y a un mort à l'étage. Est-il vraiment mort? L'ambiguïté est maintenue. J'étais dans ma maison... saisit un moment particulier de suspens entre la vie et la mort: lorsque le Jeune Frère s'est écroulé, elles ont d'abord pensé qu'il était seulement évanoui. C'est au fil de la nuit de veille qu'elles passent ensemble qu'elles prennent conscience de sa mort et commencent à évoquer leur avenir : la période de deuil, puis les perspectives de départ pour les plus jeunes. A l'ouverture elles espèrent encore qu'il s'éveillera :

La Mère. - Nous allons faire ça, oui, je passerai tout mon temps à attendre qu'il s'éveille.

Nous allons faire ça et si vous ne le faites pas, si vous ne voulez plus le faire, si celles-là ne veulent plus, si toi-même, tu m'abandonnes, si tu ne m'aides pas, oui, je le ferai seule, je resterai là, et j'attendrai seule, qu'est-ce que ça fait ? ${ }^{24}$

Comment donner à percevoir la présence-absence de ce mort qui se trouve dans la pièce du dessus ? J-L. Lagarce ne fait pas usage de la teichoscopie ${ }^{25}$. Dans J'étais dans ma maison... le Jeune Frère n'est jamais décrit « en direct " par les femmes. Fait étrange, au cours de cette nuit de veille aucune d'entre elles ne se rend dans la chambre pour surveiller le «malade» et elles acquièrent la certitude de sa mort sans avoir vu le 
cadavre. Durant ses années d'absence, elles ont fait du Jeune Frère une construction imaginaire. Elles lui ont patiemment élaboré une existence fictive : il a voyagé, il « dut livrer ses batailles, des guerres et des batailles, non? Et vainqueur, quoi d'autre ? ${ }^{26} » \mathrm{Ce}$ jeune frère est une construction mentale et c'est probablement pour cette raison qu'elles n'ont pas besoin d'aller lui prendre le pouls pour savoir qu'il est mort. Il a quitté depuis longtemps le plan du «réel». Peu importe donc que le corps soit mort. C'est « l'image » du frère ou du fils, construite durant de longues années d'attente, qui va devenir celle d'un mort. D'une certaine manière le jeune homme est encore absent, comme l'Ulysse de Elles disent..., adaptation de l'Odyssée d'Homère réalisée par J-L. Lagarce en 1975. Le dispositif annonce déjà J'étais dans ma maison... : Pénélope, Calypso, Circé et Nausicaa ont longuement attendu Ulysse. A son retour, elles disent «la vie passée dans l'attente; elles disent la solitude, elles disent l'absence. Ulysse amant, Ulysse époux, Ulysse père, Ulysse toujours absent même s'il suffit de tendre le bras pour atteindre son corps. ${ }^{27}$ » De même, le jeune homme est devenu une construction mentale, rien d'autre que la matrice de la parole qui occupe l'attente, un être déréalisé. Lorsque l'Aînée raconte son arrivée, il peut s'agir de la scène imaginée depuis des années, de la scène accomplie à laquelle elle vient d'assister à l'ouverture de la pièce, ou d'un prolongement hallucinatoire de ce moment redevenant présent. Cette ambiguïté temporelle est entretenue, tout au long de la pièce, par l'emploi successif de l'imparfait, du passé simple, du passé composé, du présent ou du futur.

L'Aînée. - Lorsqu'il passa le pas de la porte, il pose son sac,

lorsqu'il passe le pas de la porte, il pose son sac, il entre dans l'ombre de la maison, on le voit mal, je le vois mal, je le devine, à contre-jour, on le voit mal, la lumière derrière lui, j'étais sûre qu'on le verrait mal, et que ses yeux, je ne pourrais les deviner, juste la silhouette qui occupe l'entrée de la maison, et ses yeux dans l'obscurité, $[. . .]^{28}$

Ces quelques lignes contribuent à créer un effet d'incertitude quant au moment de l'énonciation; le passé simple, qui désigne un procès accompli dans le passé, est remplacé par le présent qui crée un "effet d'hallucination ${ }^{29}$ - peut-être que l'événement mentionné n'est pas tout à fait achevé, peut-être que le simple fait de le raconter permet de le revivre, dans l'univers littéral des personnages lagarciens. Dans une étude des différences entre cinéma et théâtre, Octave Mannoni pointe dans Clefs pour l'Imaginaire un phénomène particulier : "au cinéma, on dirait que l'absence de l'acteur, dans sa réalité, a pour effet de donner aux images du Moi la liberté de s'émanciper davantage.... ${ }^{30}$ " Cet effet pourrait être décuplé dans un cas de hors-scène théâtral semblable à celui qui caractérise J'étais dans ma maison.... L'absence de l'acteur contribue paradoxalement à renforcer la présence du personnage.

Dans J'étais dans ma maison... aussi, la mémoire est une mise en fiction de l'autre: les femmes ne cessent de raconter l'histoire du jeune homme en se jetant à la tête des versions contradictoires de sa dispute avec son père ou en imaginant quel a été son parcours après son départ. La fréquence des mentions des morts dans le texte crée un type particulier de présence qui est une forme de hantise. Parler des morts, c'est leur rendre un semblant d'être, une forme de présence quasi performative. L'espace scénique subit la présence-absence du cadavre si souvent mentionné dans le dialogue, il est envahi par cet élément hors-scène qui fait office de centre absent de l'espace et de l'intrigue. L'Etage est le point focal, l'instrument optique nécessaire au bon fonctionnement du dispositif et à la complétude de l'espace dramaturgique ${ }^{31}$. Concrètement, cette présence latente peut être donnée à percevoir par le jeu des 
acteurs. Ainsi, dans la mise en scène de J'étais dans ma maison... créée en 2004 par Joël Jouanneau ${ }^{32}$ le spectateur pouvait repérer le lieu supposé où se trouvait l'escalier menant à la chambre du Jeune Frère car les comédiennes le désignaient parfois d'un regard ou d'un signe de tête. Cela suffisait à donner un rayonnement particulier à une portion de l'espace située au lointain, côté jardin, sans qu'il soit nécessaire de représenter de manière réaliste un escalier. Dans le Synopsis de la pièce J-L Lagarce précise que les cinq femmes pourraient "s'appeler aussi Electre, Chrysothémis, Iphigénie, Clytemnestre et la Femme Captive, La Troyenne ${ }^{33} »$. Prenons l'exemple d'Electre: elle est le personnage hanté par excellence, le personnage construit "autour" d'un mort comme la maison tourne "autour» du jeune frère. Elle est «possédée » par un mort et lui sert de relais, comme les cinq femmes qui entretiennent le souvenir du jeune homme :

C'est à l'intérieur des vivants que s'accomplit le retour du mort ou que s'affirme la force de sa présence. Sophocle n'a-t-il pas construit tout le personnage d'Electre autour de la pensée du père mort resté vivant en elle? De toutes les forces de son être, elle proclame le refus de l'oubli et l'éternel deuil, l'éternelle mémoire née du désespoir de la mort. ${ }^{34}$

Le mort est au centre des dialogues des vivants. De l'évocation à l'invocation, la frontière devient alors floue. La fréquence, dans les dialogues des cinq femmes, des références à la chambre qui se trouve à l'étage et au jeune homme qui y repose ouvre l'espace du plateau vers ce lieu invisible. La présence d'un corps inerte, même hors scène - surtout hors scène - interroge l'essence de l'image théâtrale et la manière dont elle peut exister sur un mode autre que celui de la représentation. A la différence de Louis, le jeune homme de J'étais dans ma maison... est associé à un lieu. Il est constitué par rapport à un espace contigu au plateau auquel les autres personnages se réfèrent constamment. C'est par la parole des actrices que se dessine cette chambre: dans ce théâtre le texte est porteur de son espace de jeu, qui est d'abord performatif avant d'être figuratif. La présence latente du jeune homme mourant est perceptible parce qu'il est associé à un lieu, lieu qui tend à échapper à l'espace familier de la maison. Maurice Blanchot définit ainsi le pouvoir du cadavre à modifier quelque chose du lieu où survient la mort :

Ce qu'on appelle dépouille mortelle échappe aux catégories communes: quelque chose est là devant nous, qui n'est ni le vivant en personne, ni une réalité quelconque, ni le même que celui qui était en vie, ni un autre, ni autre chose. Ce qui est là, dans le calme absolu de ce qui a trouvé son lieu, ne réalise pourtant pas la vérité d'être pleinement ici. La mort suspend la relation avec le lieu, bien que le mort s'y appuie pesamment comme à la seule base qui lui reste. Justement, cette base manque, le lieu est en défaut, le cadavre n'est pas à sa place. [...] Le lieu où l'on meurt n'est pas un lieu quelconque. On ne transporte pas volontiers cette dépouille d'un endroit à un autre : le mort accapare sa place jalousement et il s'unit à elle jusqu'au fond, de telle sorte que l'indifférence de cette place, le fait qu'elle est pourtant une place quelconque, devient la profondeur de sa présence comme mort, devient le support de l'indifférence, l'intimité béante d'un nulle part sans différence, qu'on doit cependant situer ici. ${ }^{35}$

27 Paradoxalement, ce cadavre qu'on ne voit pas fait image. L'analogie entre l'image et la dépouille établie par Maurice Blanchot dans L'Espace littéraire peut permettre de mieux comprendre comment la présence d'un cadavre à l'étage supérieur peut agir concrètement sur ce qui se joue, hors représentation, dans l'espace scénique.

La ressemblance cadavérique est une hantise, mais le fait de hanter n'est pas la visitation irréelle de l'idéal: ce qui hante est l'inaccessible dont on ne peut se 
défaire, ce qu'on ne trouve pas et qui, à cause de cela, ne se laisse pas éviter. L'insaisissable est ce à quoi on n'échappe pas. L'image fixe est sans repos, en ce sens surtout qu'elle ne pose, n'établit rien. ${ }^{36}$ créer cette image fixe. Le lieu de la mort reste comme marqué par cet événement, qui s'est produit dans la maison où évoluent les personnages en scène, à l'étage, où l'on peut accéder par l'une des issues de la pièce où ils se trouvent. La présence du cadavre tend à envahir le lieu, et c'est précisément ce processus qui est au coeur de l'intrigue de J'étais dans ma maison....

On le sait, «à un certain moment », la puissance de la mort fait qu'elle ne s'en tient plus au bel endroit qu'on lui a assigné. Le cadavre a beau être étendu tranquillement sur son lit de parade, il est aussi partout dans la chambre, dans la maison. ${ }^{37}$

Si le cadavre est image, c'est en tant qu'il renvoie à l'absence de la chose tout en faisant de cette absence une forme de présence. ${ }^{38}$ Le rapport à l'image du mort - ou du mourant - qui s'établit est d'ordre iconique : l'image iconique a pour fonction d'établir une relation, d'orienter le regard. Ce qui se joue dans la pièce étudiée ici s'apparente au passage du cadavre à l'image décrit par M. Blanchot dans L'Espace littéraire. Ainsi, dans J'étais dans ma maison... la confusion entre l'agonie du jeune homme et le sommeil de l'enfant est toujours maintenue car la nuit de veille des cinq femmes constitue ce moment ambigu de transition vers l'image cadavérique. La présence d'un étage horsscène produit ici un effet d'ordre iconique : il n'est pas image, mais relation. Dans J'étais dans ma maison... le sujet absent est associé à un lieu et doit une part de sa présence paradoxale à cette spatialisation : lorsque les femmes désignent l'espace de la chambre, lorsque cette chambre se construit peu à peu par le biais des déictiques, s'établit un rapport d'équivalence entre le personnage et la portion d'espace qui lui est assignée.

Le sujet-personnage du Jeune Frère devient dans J'étais dans ma maison... objet de discours, objet d'attente. Bien qu'il n'entre jamais en scène, il ne disparaît pas, au contraire: il acquiert une présence d'une intensité particulière. Son parcours reste voué à l'incertitude : dans les trois textes étudiés ici le sujet au sens psychologique du terme apparaît inconnaissable. Cependant, les personnages lagarciens ne peuvent être considérés uniquement comme des simulacres de personnes. La notion de sujet peut prendre un sens psychologique dans ce théâtre, mais les êtres qui évoluent en scène ne font pas toujours illusion: mi-acteurs, mi-récitants, ils semblent incarner un souvenir du personnage au sens traditionnel du terme.

\section{Des personnages entre présence et absence}

Qu'est-ce qu'un personnage de théâtre ? Il est incarné de manière toujours provisoire dans un corps qui n'est pas le sien, il n'a d'existence que sur scène et pendant la durée de la représentation, c'est un sujet inventé de toutes pièces et recréé chaque soir par un comédien. Le théâtre de J-L. Lagarce joue des multiples facettes de la notion. Le personnage dramatique est un sujet polymorphe à la définition problématique. Robert Abirached constate dans son introduction à La Crise du personnage dans le théâtre moderne que les différents sens donnés à la notion de personnage permettent, malgré leurs contradictions, d'esquisser une "structure permanente» du personnage dramatique. « Toutes ces acceptions ont ceci de commun qu'elles indiquent une mise en rapport de l'homme réel avec des images de lui agrandies ou exemplaires, obtenues par imitation 
et soumises à reconnaissance. ${ }^{39}$ " $\mathrm{Si}$ R. Abirached préfère le terme de "structure " à celui de " définition ", c'est probablement pour souligner le caractère problématique de la notion, qui résiste à l'analyse. Il repère également deux grands moments de rupture : la «crise de la représentation » de la fin du XIXe siècle, puis l'avènement des théories de Craig, de Brecht et d'Artaud, à la suite desquelles les notions de mimésis et de représentation ont fait l'objet d'une remise en cause radicale entraînant une modification importante du statut du personnage dramatique. Le théâtre de J-L. Lagarce, postérieur à ces bouleversements, en a subi l'influence. Que devient le personnage dramatique à la fin du XXe siècle? Au terme de personnage, Julie Sermon préfère celui de "figure ", qui lui semble mieux correspondre au statut des êtres en scène de certaines dramaturgies contemporaines. Le personnage contemporain tend à s'éloigner de la structure définie par R. Abirached pour devenir un être " à construire ", selon la formule de Jean-Pierre Sarrazac :

La figure ne représente donc ni l'hypostase ni la dissolution mais un nouveau statut $\mathrm{du}$ personnage dramatique : personnage incomplet et discordant qui en appelle au spectateur pour prendre forme ; personnage à construire..$^{40}$

Cela implique une participation du spectateur, confronté à un être qui n'est pas donné mais à obtenir. Ce personnage incomplet, né du passage d'une dramaturgie de l'action à une dramaturgie de la parole, ne fait pas nécessairement corps avec ce qu'il énonce.

L'affaiblissement du personnage énonciateur, sa démultiplication ou sa suppression pure et simple est une autre modification notable. La parole n'est plus nécessairement énoncée par un personnage construit, à l'identité repérable. Ca parle toujours mais on ne sait pas toujours d'où ça vient, faute de repères sociaux, psychologiques, ou simplement d'identité affichée. [...] Les relations entre la parole et l'action, contradictoires ou divergentes, donnent à voir le trouble ou les stratégies de personnages qui ne correspondent pas fatalement à ce qu'ils disent ou à ce qu'ils font. Tout un théâtre est construit strictement sur le terrain de la parole, comme si les véritables enjeux se situaient dans les défis et les fragilités de son émergence, que la parole était seule apte à construire une réalité théâtrale qui se défie des conventions. ${ }^{41}$

L'énonciation est bien souvent au centre du drame chez J-L. Lagarce. Les personnages se caractérisent par un "mal à dire " qui contribue à laisser dans l'ombre certains aspects de leur identité. Ils peinent à raconter " l'histoire d'avant ${ }^{42}$, leurs souvenirs manquent parfois de précision et leur passé est par conséquent toujours un objet d'incertitude. S'inspirant de l'effet-personnage théorisé par Vincent Jouve, Julie Sermon propose d'employer le terme d'effet-figure pour désigner le vide ontologique qui caractérise le personnage contemporain :

[...] l'effet-figure est d'abord celui d'un travail de l'entre-deux (de la proximité et de la distance, de l'illusion et de son déni), qui estompe les contours stables du personnage, trouble les catégories de prime abord établies, et finalement, tient les événements comme les identités en suspens de l'être-là théâtral : les figures ont une ontologie flottante, entre acteur et personnage, réalité et fiction - état de présence équivoque qui questionne l'imaginaire même de la représentation [...] si le personnage peut être un rouage narratif, rester un support à projection, il est aussi l'endroit par où se voit piégée, détournée, mise à mal l'illusion. ${ }^{43}$

La figure n'est cependant pas une négation ou une annulation du personnage :

la figure n'est pas un non-personnage, elle est son autre - elle le trouble et le déjoue dans son fonctionnement même, ne cristallise rien. [...] elle serait plutôt de l'ordre d'une force - un principe d'action et de déformation - une énergie qui travaille à modifier un équilibre ${ }^{44}$ 
J'ai fait l'hypothèse que l'effet-figure dans le théâtre de J-L. Lagarce tend à reléguer hors scène le personnage au sens traditionnel du terme: sur le plateau, restent des êtres au satut incertain qui semblent convoqués là pour rejouer une histoire déjà vécue. Ce sont les créatures en scène qui assurent alors la "mise en représentation $»^{45} \mathrm{du}$ personnage. On ne saura presque rien de leur passé, d'ailleurs ce sont peut-être des acteurs... Juste la fin du monde, J'étais dans ma maison et j'attendais que la pluie vienne et Le Pays lointain mettent en jeu des personnages paradoxaux à la présence ambiguë; le personnage lagarcien y apparaît travaillé par un devenir figure dont le mouvement est particulièrement net dans Le Pays lointain. Elles permettent d'explorer plusieurs modalités d'absence du personnage au sens traditionnel du terme.

\section{Personnages paradoxaux}

Des personnages paradoxaux sont introduits dans Le Pays lointain: les plus aisément repérables pour le lecteur sont les deux morts (L'Amant, mort déjà et Le Père, mort déjà) et les deux entités collectives constituées par Le Garçon, tous les garçons et Le Guerrier, tous les guerriers, "dont la désignation révèle bien qu'ils ne valent pas sur scène en tant que sujets, mais qu'ils ont au contraire un fonctionnement générique ${ }^{46} »$. Ils ne se situent à première vue pas sur le même plan que les autres personnages, plus proches du modèle de la personne. Ils s'en distinguent de manière souvent ténue : les morts restent des commentateurs distants des retrouvailles, ils ne peuvent pas agir au même titre que les autres. Cependant ils peuvent communiquer avec les autres personnages, et des rencontres ont lieu : ils ne sont pas là uniquement pour raconter l'histoire de Louis, ils ont aussi une réelle singularité. Cela devient perceptible si on s'intéresse aux interactions entre les deux familles. En effet, Louis s'est rendu dans sa famille réelle accompagné du seul Longue Date. Pourtant, il est accompagné des personnages de la famille inventée qui communiquent parfois avec les autres, avec ceux qui sont présents à part entière ou plutôt présents sur un mode « réaliste ». Hélène fait partie de ces personnages à la présence ambiguë. Elle peut parler à Catherine et à Suzanne alors qu'elle n'est pas censée être là. Nous l'apprenons sur le mode du « flashback » : elle n'est pas venue avec Louis et Longue Date.

Hélène. - Et je pourrai dormir à l'hôtel, je pouvais dormir à l'hôtel, je serai dans la même ville, cette sorte de ville...

Longue Date. - On en a déjà parlé. La Femme qui accompagne l'Homme qui accompagne l'Homme qui revient voir ses parents. Un groupe. Une délégation.

Hélène. - Tu me téléphoneras.

Longue Date. - Je te téléphonerai.

L'Amant, mort déjà, pause sa main sur l'épaule d'Hélène.

Hélène. - Après, je n'ai rien fait, je suis restée là comme je suis toujours. J'ai attendu. ${ }^{47}$

Rappelons-le une fois encore : le théâtre de J-L. Lagarce est pensé en vue d'un devenir scénique. C'est parce que nous sommes au théâtre que ces rencontres entre personnages absents sont possibles. Entre personnages, ou plutôt entre figures : cette notion forgée par Julie Sermon est plus adéquate à la description des êtres en scène 
lagarciens. Le personnage est relégué ailleurs. Il y a l'idée du personnage traditionnel, mais ce n'est pas cela que nous voyons en scène. Ainsi du Père, mort déjà, qui n'est plus tout à fait lui-même et qui se présente à l'Amant en disant : " Je suis son père. J'étais. ${ }^{48}$ " Les morts entrent en scène dans Le Pays lointain, ils quittent l'étage mais ce ne sont pas des fantômes. Ils ne peuvent pas dire "je suis » mais ils sont là, à leur manière. Il semble que ces figures paradoxales aient différentes manières d'intervenir, différentes modalités de présence aussi :

L'Amant, mort déjà. - Je regarde. Je ne bouge pas. Je n'interviens pas. Je n'en ai pas

les moyens. Je me pose par là, je m'assieds.

Le Père, Mort déjà. - Je reste près de vous et je regarde aussi. ${ }^{49}$ référence à des codes. La pièce représentée sur scène semble parfois en cours de répétition, et certaines répliques sont entrecoupées de remarques qui pourraient être les commentaires d'un acteur travaillant son rôle.

L'Amant, mort déjà - Et la Famille qu'on voulut se choisir, la famille secrète, l'Autre Famille, celle-là qui parfois ne sait même pas qu'on se la construisit sans bruit.

Longue Date - Hélène?

Hélène - Oui ?

Longue Date - C'est à toi.

Hélène - Pardon. Excusez-moi. Je l'écoutais celui-là, toujours j'aime à l'écouter. ${ }^{51}$

41 Ce glissement du personnage vers l'acteur est particulièrement perceptible chez « un Garçon, tous les garçons » et « le Guerrier, tous les guerriers » :

Le Guerrier, tous les guerriers. - Le Guerrier, tous les guerriers. Tous ceux-là que je fais, ceux-là que je joue, mon groupe entier, ceux-là qui vivaient comme moi, ceuxlà qui sont toujours solitaires et le croisèrent, croisèrent Louis, le croisèrent et ne voulurent laisser aucune trace, eurent bien trop peur de s'attacher à lui, et de perdre pied et de s'éprendre et souffrir..$^{52}$ 
Le phénomène est encore plus clair dans le cas du Garçon, qui fait explicitement référence au travail de mémorisation de l'acteur :

Un Garçon, tous les garçons. - Un Garçon, tous les garçons.

J'énumère, $\mathrm{j}$ 'essaie de me souvenir et j'énumère. La liste là de tous les personnages que je joue.

J'ai appris tout cela par coeur, c'est du travail..$^{53}$

Ainsi le drame semble-t-il préexister à sa " mise en représentation ». Les personnages semblent convoqués pour jouer un rôle, mais ils jouent parfois avec tant de sérieux que leur nature d'acteurs peut être mise en doute. C'est pourquoi il s'agit seulement d'un glissement vers l'acteur. Le statut indéterminable de ces êtres suspendus entre le personnage et l'acteur est caractéristique de ce que Julie Sermon nomme «l'entredeux $\|^{54}$ lagarcien. Dans Le Pays lointain, rien ne permet de déterminer si le drame est rejoué par des acteurs ou " vécu " par des personnages.

Louis prend parfois le statut de metteur en scène par rapport au Garçon et certains de leurs échanges s'apparentent à des dialogues acteur-metteur en scène.

Un Garçon, tous les garçons. - Je ne sais plus avec exactitude, on note mal, tu notes mal, tu as écrit ça, « un gigolo efficace ", mais j'imagine que tu pensas plus qu'il en avait l'allure, l'allure et la manière, le comportement, mais je ne suis pas certain que tu crus qu'il en avait l'emploi.

On fera la scène sans argent.

Louis. - Je ne suis pas certain qu'on fera la scène..$^{55}$

$\mathrm{Au}$ fil de cette scène située dans la deuxième partie de la pièce le Garçon énumère ses différents rôles tout en les ponctuant de commentaires comme « Rôle silencieux ${ }^{56}$ ou «Si quelqu'un d'autre veut faire le boxeur à ma place, je n'en prendrai pas ombrage, je ne me sens pas de taille. ${ }^{57}$ » De temps à autres Louis donne quelques indications. Le Garçon fait parfois des suggestions. Louis, bien qu'il endosse parfois le rôle du metteur en scène, n'a pas une maîtrise totale de ses « acteurs » ni des événements de son passé. Dans Juste la fin du monde l'indétermination de l'identité de Louis était entretenue par sa propension à faire et refaire sans cesse le récit de son passé. Ce phénomène persiste dans Le Pays lointain, amplifié par l'hésitation maintenue entre intersubjectivité et intrasubjectivité, entre jeu et "réalité ». Reste que l'esquisse d'un Louis metteur en scène peut amener à poser la question d'une possible émergence du sujet-auteur. Il ne m'a pas semblé nécessaire de revenir sur la complexité du brouillage des données biographiques à l'oeuvre dans les textes étudiés : Jean-Pierre Thibaudat a déjà montré que l'hypothèse d'une identification de Louis à J-L Lagarce et de certains des personnages à ses proches ne résiste pas à une lecture attentive du texte. ${ }^{58} \mathrm{Ce}$ théâtre intime n'est pas celui de l'intimité de J-L. Lagarce. Reste que la présence d'une figure de metteur en scène y est suggérée, et que le texte semble travaillé par le point de vue d'un tiers qui observe et qui choisit les modalités de la représentation. Dans l'espace du hors-scène, se dessinent une pratique de l'écriture ${ }^{59}$ et un regard de metteur en scène qui marquent la présence d'un sujet double, auteur et praticien du théâtre, hypothétique point de vue sur un texte dramatique et sur ses modes de représentation. Instance vouée à la fabrique de l'oeuvre, ce sujet-là ne peut - et ne doit - pas être confondu avec l'auteur en tant que personne. En revanche, la suggestion de l'existence $\mathrm{du}$ point de vue d'un tiers potentiellement metteur en scène qui assisterait à la représentation me semble être l'une des manifestations du hors-scène lagarcien : plus 
qu'un sujet, c'est un regard que le statut assumé d'acteurs des figures lagarciennes permet de repérer, parce qu'il leur sert de point de référence.

\section{Le personnage mis hors-scène}

De Juste la fin du monde au Pays lointain, le statut des personnages se complexifie. Les personnages-récitants de la première version deviennent acteurs ou metteurs en scène. Les membres de la famille de Louis semblent spécialement convoqués sur scène pour rejouer son histoire, tels des acteurs qui demandent: "On se met où? " lorsqu'ils travaillent leur entrée. Cependant ils semblent par moments reprendre leur statut ordinaire de personnages dramatiques. Selon Julie Sermon la dramaturgie du Pays lointain ne repose que sur cet entre-deux : « configurer et mettre en scène en temps réel et en personne le champ de la fiction dramatique $»^{60}$. Les personnages sont à la fois " acteurs et récitants, maitres d'oeuvre et ouvriers d'événements qui ne se jouent qu'au second degré ${ }^{61}$ ». Le sujet lagarcien, c'est d'abord le sujet parlant, et donc l'acteur, condition de l'esquisse des autres manifestations du sujet dans cette dramaturgie. C'est lui qui assure la mise « hors-scène » du personnage.

Pour esquisser une définition plurielle du personnage lagarcien, il faudrait commencer par rappeler qu'il se caractérise par une langue particulière, toujours utilisée au second degré. Dans Juste la fin du monde et dans J'étais dans ma maison..., les personnages lagarciens tirent leur spécificité de leur rapport au langage. Le personnage est ici l'archétype du locuteur ironique tel que le définit Oswald Ducrot dans Le dire et le dit ${ }^{62}$ : ce locuteur met en scène un énonciateur dont il ne prend pas en charge le discours pour donner à percevoir l'ironie de son propos. Cette théorie peut être éclairante dans la mesure où elle présuppose un dédoublement du sujet. Or, le personnage lagarcien se construit en fonction d'une logique de dédoublement induite par son langage. L'impuissance de Louis à raconter son histoire implique une mise en doute généralisée de la parole et de son authenticité. Dans les trois pièces étudiées cette logique de dédoublement peut s'appliquer au drame lui-même, drame toujours précédé par un drame premier, et pourtant toujours vécu dans «l'ici et maintenant» de la représentation. Peut-être le " personnage » lagarcien ne peut-il porter ce nom que dans ce drame hors-scène qui fait l'objet à la fois d'un récit et d'une reconstitution sur le mode du jeu de rôles. Chez J-L. Lagarce, le personnage au sens traditionnel du terme est toujours cantonné dans un ailleurs de l'espace scénique. Car les figures inaugurent une manière d'être en scène qui fait problème.

Il y a une présence propre à la figure, présence que Julie Sermon estime de type performatif :

L'espace-temps de la figure est donc celui de la présence immédiate, du cadrage instantané, et dédramatisé. C'est pourquoi, en reprenant les termes de Gilles Deleuze [...], je dirais que par contraste avec le personnage, le rapport que les figures entretiennent à la représentation n'est plus «figuratif » mais « figural »: personnages d'un théâtre désinstrumentalisé, leur corps n'est ni illustratif, ni narratif, mais performatif. Sur scène, les figures ne représentent rien; elles s'imposent comme force d'apparition par la parole, et ne donnent rien d'autre à dire que cet événement poïetique-là. ${ }^{63}$

La figure semble pourtant jouer de nos habitudes de spectateurs, s'attendant encore à voir au théâtre des personnages. C'est ce décalage, ou du moins ce temps d'adaptation nécessaire au regard du spectateur, qui amène une interrogation sur la présence 
véritable de ces êtres fragmentaires ou paradoxaux, souvent habités par un passé horsscène inconnaissable. Bien sûr, les figures sont présentes puisque les acteurs sont là. Mais justement, ce n'est pas une présence de l'ici et maintenant; il y a déplacement, dans le lieu scénique consacré au présent, de créatures qui viennent d'ailleurs et de loin et qui peuvent être ailleurs et loin tout en étant là. Le théâtre de J-L. Lagarce pose avec acuité la question de la présence, qui me semble intrinsèquement liée au hors-scène : les figures de ses textes entrent en scène avec pour arrière-plan le personnage dans sa conception traditionnelle et sa dramaturgie semble jouer des habitudes du spectateur, déjouant ses attentes et reléguant hors-scène ce qui semble être un hypothétique drame absolu au sens szondien ${ }^{64}$ du terme. A travers le mouvement vers un passé perdu, vers la portion hors-scène de l'existence des personnages, on peut se demander si la fable au sens traditionnel du terme n'est pas un horizon (inaccessible) du horsscène lagarcien. La fable persiste dans cette dramaturgie sous une forme déstructurée et morcelée, et correspond au sens « contemporain » que lui donne J-P. Ryngaert :

Souvent mise en question, la fable survit au moins sous la forme de fragments de récits ou d'assemblage d'événements dont l'importance est difficile à mesurer. On parle souvent de "micro-fables" correspondant à des récits minimaux ou fragmentaires, ou de « fables ambiguës » quand elles se prêtent à un grand nombre d'interprétations. ${ }^{65}$

Le Pays lointain, notamment, donne à supposer l'existence d'une continuité perdue de la fable, alors fragmentée entre scène et hors-scène mais non supprimée, comme le remarque Françoise Dubor. "L'unité dramaturgique absente de l'écriture est à la fois désignée et reléguée dans le passé, désignée révolue, dans le mouvement général de révolution que traverse Louis pour accomplir son retour. ${ }^{66}$ » L'histoire à recomposer est à la fois présente de par l'obsession du passé dont font preuve les personnages et maintenue au rang d'un hypothèse incertaine : le lecteur-spectateur est alors confronté à l'impossibilité de choisir un point de vue sur ce passé. Reste ce vague souvenir d'une fable inaccessible, dans laquelle on aurait pu parler de personnage et dans laquelle la définition du sujet aurait peut-être été moins problématique.

\section{Conclusion}

51 Dans la dramaturgie lagarcienne le sujet, lorsqu'il est mis hors-scène, demeure indécidable. Les identités se donnent à construire pour mettre plus que jamais le spectateur en jeu. Peut-être s'agit-il de ces «nouvelles invisibilités» définies par M-J. Mondzain. Car les « images » du hors-scène lagarcien sont des objets de perception qui résistent à toute réduction, à tout enfermement dans un point de vue unique; de plus leur relation à la parole des personnages est fondamentale. Elles proposent une alternative au spectaculaire ambiant qui pourrait être décrite ainsi :

L'anéantissement de l'imaginaire du visible ne peut que relancer, plus violemment que jamais, la revendication existentielle de la parole ; c'est elle qui garantit la présence de l'énigme, c'est-à-dire que c'est elle qui insuffle au visible la forme de nouvelles invisibilités. A présent, tout peut se voir et la terre entière se donne en spectacle à elle-même. Tous les penseurs et les créateurs doivent inventer dans ce nouvel espace l'émergence de lieux où l'image se love et nous attend pour se manifester. ${ }^{67}$

52 C'est au niveau de la parole des personnages que se situe le véritable événement dans ce théâtre : dire quelque chose du passé, essayer de raconter son histoire, confronter différentes versions permet de mettre littéralement en présence ces invisibilités. Le 
hors-scène lagarcien peut être qualifié de chronotope, dans la mesure où il est fréquemment situé dans le temps avant de l'être dans l'espace :

Le seuil est une ligne imaginaire faisant office de séparateur, mais aussi de transition pour l'espace comme pour le temps, et entre l'espace et le temps. [...] Qu'il soit temporel ou spatial, dans cet univers de l'attente le chronotope prend la forme d'un seuil. Le jeune homme vient de franchir le seuil dans l'autre sens, de l'extérieur vers l'intérieur, du monde extérieur vers l'origine. ${ }^{68}$

53 Le sujet lagarcien, parce qu'il se constitue fondamentalement entre deux espaces, me semble pouvoir être qualifié de sujet «du seuil»: en scène, lorsqu'il est acteur ou narrateur, il peut être projeté ailleurs, associé à un lieu précis ou diffracté dans un hors-scène plus temporel que spatial. Cet effet de «mise en présence » du passé est une fonction essentielle du hors-scène lagarcien et contribue à l'élaboration d'une temporalité propre à cette dramaturgie. On retrouve l'obsession du présent que J-P. Ryngaert considère comme caractéristique de la dramaturgie contemporaine :

L'« ici et maintenant » du théâtre devient le creuset où le dramaturge conjugue à tous les temps les fragments d'une réalité complexe, où les personnages, saisis par l'ubiquité, voyagent dans l'espace du rêve ou bien, davantage encore, par le travail de la mémoire. ${ }^{69}$

54 C'est un présent «théâtral » que crée l'écriture lagarcienne, qui instaure une temporalité du jeu caractérisée par un mouvement de répétition-variation : "comme une esquisse de ce que cela aurait pu être ou de ce que cela fut » : toute représentation ne se définit-elle pas dans cet entre-deux, à la fois répétition et moment unique ? $\mathrm{Ce}$ passé hors-scène dont il a été si souvent question au cours de cette recherche n'est-il pas alors, d'une certaine manière, remis "en scène »? Car dans les textes du corpus il est impossible de savoir à quel moment le passé narré est remis en jeu, par le fait même d'en parler, et par les oppositions ou mouvements communs que rencontre cette parole chez les autres personnages. En 1995, lors d'un entretien avec Lucien Attoun, J-L. Lagarce formulait en ces termes cette idée du théâtre comme répétition :

Je ne peux pas m'empêcher de considérer ce qui a lieu sur la scène comme ayant

déjà eu lieu, comme étant répété, comme ayant déjà été entendu. ${ }^{70}$

Cette conception de la représentation comme réitération de quelque chose qui aurait déjà eu lieu est à l'oeuvre dans les trois textes étudiés : impossible de savoir si le retour de Louis dans Le Pays lointain n'est pas une simple reconstitution. La temporalité est ainsi brouillée. Ces textes entretiennent une indécision quant à la nature de la représentation, qui ne se donne toujours déjà comme répétition. Peut-être le drame déjà joué est-il seulement commenté par les êtres en scène, peut-être est-ce cela, la représentation? Mais pourquoi semblent-ils parfois investis par leur personnage ? Si les textes de J-L. Lagarce relèvent d'un théâtre épique, cela ne semble pas impliquer une rupture radicale avec l'idée d'un théâtre dramatique qui subsiste comme arrièreplan ou comme souvenir. C'est une écriture traversée par la conscience d'être précédée par d'autres écritures et par une conception du théatre comme drame avec laquelle elle ne cesse de jouer. Aussi pourrait-on considérer que le hors-scène des textes du corpus est bien souvent porteur de l'idée d'un drame au sens szondien du terme, souvenir d'une ancienne unité de la fable désormais morcelée et jouée sur le mode ambigu de la reconstitution d'une pièce ancienne et inaccessible - d'un répété hors-scène. De ce point de vue le théâtre de J-L. Lagarce me semble pouvoir être qualifié de postdramatique car le drame, remis en question dans son unité par cette écriture très narrative et par la fragmentation de la fable, y subsiste comme souvenir ou arrière- 
plan. Comme le précise H-T. Lehmann, "postdramatique " ne signifie pas "négation abstraite, ignorance pure et simple de la tradition du drame $»^{71}$. Le drame, affaibli, se maintient dans ce théâtre « comme attente d'une grande partie de son public, comme base de nombreuses de ses formes de représentation, en tant que norme de dramaturgie fonctionnant automatiquement $»^{72}$. Rappelant que Heiner Müller a intitulé son texte postdramatique "Bildbeschreibung ", " un paysage au-delà de la mort » et "explosion d'un souvenir dans une structure dramatique morte", H-T. Lehmann commente ainsi cette formule :

On peut décrire ainsi le théâtre postdramatique : les membres ou les branches de l'organisme dramatique sont, même comme matériau moribond, toujours présents, et continuent l'espace d'un souvenir qui éclate et «s'éclate » à la fois. Même le préfixe "post-» dans le terme postmoderne, où il représente davantage qu'un simple jeton, indique qu'une culture ou une pratique artistique a transgressé l'horizon de la modernité jusqu'ici communément admis mais qui subsiste néanmoins toujours dans un quelconque rapport - négation, déclaration de guerre, libération, peut-être seulement même déviation et gai savoir de ce qui serait possible au-delà de cet horizon. ${ }^{73}$

56 L'idée d'un théâtre porteur d'un souvenir éclaté du drame me semble utile à la compréhension du hors-scène lagarcien, en tant qu'il situe la représentation comme récit / remise en jeu au présent d'un drame ancien et déjà joué. D'autre part, les textes de J-L. Lagarce permettent ainsi de penser les rapports de la représentation au réel en échappant à une problématique de l'illusion et à l'opposition binaire réalité / fiction :

Les mondes où les figures apparaissent forment un tout, cohérent, mais qui ne s'impose pas comme reproduction illusoire de réalité : ils ouvrent plutôt un espace de jeu du réel - c'est-à-dire : un espace-temps « à côté ", potentiel ${ }^{74}$, où les données de la représentation sont soumises à d'autres lois, obéissent à d'autres mouvements, donnent lieu à de nouvelles expérimentations imagées. ${ }^{75}$

Ce phénomène remet en question le second niveau de la définition du hors-scène proposée par Patrice Pavis dans son Dictionnaire du théâtre : en effet, il y est précisé que «le statut du hors-scène varie selon le degré de réalité que le milieu scénique prétend avoir ${ }^{76}$ ». Dans les textes de J-L. Lagarce ce "degré » ne peut être évalué avec certitude. L'indécidabilité du lieu scénique lagarcien oblige à repenser la dichotomie "représentation naturaliste» / "spectacle limité à l'aire de jeu " ${ }^{77}$ pour créer un espace autre. Ainsi, le statut du hors-scène se donne toujours à construire, à interpréter. De ce point de vue le hors-scène du théâtre de J-L. Lagarce accorde une place primordiale au spectateur, sujet remis au centre de la relation théatrale dans cette dramaturgie.

S'il y a un hors-scène spécifique aux écritures contemporaines, il serait à situer du côté de la recherche de présence dont H-T. Lehmann souligne l'importance parmi les formes les plus récentes du théâtre postdramatique :

Face au modèle de la représentation se trouve l'idéal utopique de la "présence » radicalisé dans le théâtre postdramatique. Lorsque, quant à la présence théâtrale en soi, Claude Régy constate avec raisons qu'elle serait «si forte dans sa simplicité qu'elle en est inaperçue ", alors, le théâtre postdramatique cherche par tous les moyens à rendre consciente cette présence. ${ }^{78}$

59 La mise hors-scène du sujet - avec ses multiples sens, déjà évoqués - me semble avoir pour effet de créer cette présence particulière à une dramaturgie qui interroge fondamentalement la nature de la représentation théâtrale. 


\section{BIBLIOGRAPHIE}

Lagarce, Jean-Luc, Music-hall, Juste la fin du monde, in Théâtre complet III, Besançon, Les Solitaires Intempestifs, 1999.

Lagarce, Jean-Luc, J'étais dans ma maison et j'attendais que la pluie vienne, Le Pays lointain, in Théâtre complet IV, Besançon, Les Solitaires Intempestifs, 2002.

Lagarce, Jean-Luc, J'étais dans ma maison et j'attendais que la pluis vienne, Paris, Théâtre Ouvert, tapuscrit 81, 1995.

Problématiques d'une oeuvre, actes de colloque (18-20 oct. 2006, Théâtre National de Strasbourg et Université Marc Bloch - Strasbourg II, dir. G. Jolly, D. Juillard, A. Pascaud), Besançon, Les Solitaires Intempestifs, coll. « Colloques année (...) Lagarce », janvier 2007.

Abirached, Robert, La Crise du personnage dans le théâtre moderne, Paris, Gallimard, Tell, 1994, (1ere éd. : 1978).

Bazin, André, " Théâtre et cinéma », in Qu'est-ce que le cinéma ?, Paris, Les Editions du Cerf, coll. 7e art, 1987.

Blanchot, Maurice, L'Espace littéraire, Paris, Gallimard, coll. Folio Essais, 1955.

Borie, Monique, Le Fantôme ou le théâtre qui doute, Arles, Actes Sud / Académie expérimentale des théâtres, coll. « Le temps du théâtre » (dir. Georges Banu), 1997.

Didi-Huberman, Georges, Devant l'image, Paris, Les Editions de Minuit, coll. « critique » (dir. Jean Piel), 1990.

Ducrot, Oswald, Le dire et le dit, Paris, Ed. de Minuit, 1984.

Issacharoff, Michael, Le Spectacle du discours, Paris, José Corti, 1985.

Lehmann, Hans-Thies, Le Théâtre postdramatique, Paris, L'Arche, septembre 2002.

Mannoni, Octave, Clefs pour l'Imaginaire ou l'Autre Scène, Paris, Seuil, Points, 1969.

Pavis, Patrice, Dictionnaire du théâtre, préf. Anne Ubersfeld, Paris, Dunod, 1996.

Pavis, Patrice, Le théâtre contemporain, Analyse des textes, de Sarraute à Vinaver, Paris, Nathan, coll. Lettres sup, 2002.

Rykner, Arnaud, Paroles perdues, Faillite du langage et représentation, Paris, José Corti, coll. Les Essais, oct. 2000.

Ryngaert, Jean-Pierre, Lire le théâtre contemporain, Paris, Armand Colin, coll. Lettres sup., (1ère éd. : Paris, Dunod, 1993), 2005.

Sarrazac, Jean-Pierre, L'Avenir du drame, Belfort, Circé, coll. Poche, février 1999.

Sermon, Julie, L'effet-figure : états troublés du personnage contemporain (Jean-Luc Lagarce, Philippe Minyana, Valère Novarina, Noëlle Renaude), thèse, sous la direction de Jean-Pierre Ryngaert, Université Paris 3 - Sorbonne Nouvelle, soutenance le 22 novembre 2004.

Szondi, Peter, Théorie du dame moderne, Belfort, Circé, coll. Penser le théâtre, 2006 (1ère éd. Frankfurt und Main, Suhrkamp Verlag, 1965).

Thibaudat, Jean-Pierre, Le Roman de Jean-Luc Lagarce, Besançon, Les Solitaires Intempestifs, 2007. 


\section{NOTES}

1. Sur cette question, on peut se référer à la thèse de Julie Sermon : L'Effet-figure : états troublés du personnage contemporain, (Jean-Luc Lagarce, Philippe Minyana, Valère Novarina, Noëlle Renaude), sous la direction de Jean-Pierre Ryngaert, Université Paris 3 - Sorbonne Nouvelle (soutenue le 22 novembre 2004) ou à l'article "L'entre-deux lagarcien : le personnage en état d'incertitude ", in Problématiques d'une oeuvre, Besançon, Les Solitaires Intempestifs, 2007, p. 59-78.

2. L'expression est de Jean-Pierre Ryngaert. Voir Ryngaert, Jean-Pierre, Lire le théâtre contemporain, Paris : Armand Colin, coll. Lettres sup., pp. 102-103.

3. Didi-Huberman, Georges, Devant l'image, Paris, Les Editions de Minuit, coll. Critique, 1990, p. 26.

4. Ibid, p. 26.

5. Lagarce, Jean-Luc, Music-hall, in Théâtre complet III, Besançon, Les Solitaires Intempestifs, 1999, (p. 57-102), p. 97.

6. Lagarce, Jean-Luc, Juste la fin du monde, in Théâtre complet III, (p. 203-280), pp. 207-208.

7. Abirached, Robert, titre de l'introduction de La Crise du personnage dans le théâtre moderne, Paris, Gallimard, Tell, 1994, p. 7.

8. Sermon, Julie, «L'entre-deux lagarcien : le personnage en état d'incertitude », art. cit., p. 61.

9. Lagarce, Jean-Luc, Juste la fin du monde, op.cit., pp. 207-208.

10. Ibid., p. 207-208.

11. Ibid., p. 234.

12. Bazin, André, "Théâtre et cinéma ", in Qu'est-ce que le cinéma?, Paris, Les Editions du Cerf, coll. 7e art (dir. Guy Hennebelle), 1987, p. 160.

13. Lagarce, Jean-Luc, Juste la fin du monde, op.cit., p. 236

14. Lagarce, Jean-Luc, Le Pays lointain, in Théâtre complet IV, Besançon, Les Solitaires Intempestifs, 2002, (p. 273-419), p. 277.

15. Ibid., p. 393.

16. Abirached, Robert, op.cit., p. 203.

17. Lagarce, Jean-Luc, Le Pays lointain, op.cit., p. 281.

18. Saint-Pol Roux, Les Personnages de l'individu, in Le Tragique dans l'homme I, Monodrames, Mézières-sur-Issoire, éd. Rougerie, 1983.

19. Lagarce, Jean-Luc, Le Pays lointain, op.cit., p. 315.

20. Ibid., p. 321.

21. Rykner, Arnaud, Paroles perdues, Faillite du langage et représentation, Paris, José Corti, coll. Les Essais, 2000, p. 76.

22. Lagarce, Jean-Luc, Le Pays lointain, op. cit., p. 234.

23. Ibid., p. 231.

24. Ibid., p. 235.

25. Teichoscopie: «moyen dramaturgique pour faire décrire par un personnage ce qui se passe en coulisses dans l'instant même où l'observateur en fait le récit (hors-scène). », in Pavis, Patrice, Dictionnaire du théâtre, préf. Anne Ubersfeld, Paris : Dunod, 1996, article « teichoscopie », p. 346.

26. Ibid., p. 234.

27. Texte du programme des « Rencontres amateur » de mai 1975 (Besançon), cité par Jean-Pierre Thibaudat in Le roman de Jean-Luc Lagarce, Besançon, Les Solitaires Intempestifs, 2007, p. 38.

28. Lagarce, Jean-Luc, Le Pays lointain, op. cit., p. 235.

29. L'expression est employée par Patrice Pavis in Le théâtre contemporain, Analyse des textes, de Sarraute à Vinaver, Paris, Nathan, coll. Lettres sup, 2002, p. 187.

30. Mannoni, Octave, Clefs pour l'Imaginaire ou l'Autre Scène, Paris, Seuil, Points, 1969, p. 180.

31. La notion d'espace dramaturgique est empruntée à $M$. Issacharoff et désigne "l'emploi particulier du lieu scénique » par le dramaturge. L'espace dramaturgique comprend un espace 
mimétique et un espace diégétique (hors-scène). Voir Issacharoff, Michael, Le Spectacle du discours, Paris, José Corti, 1985, p. 69.

32. Spectacle créé le 5 juillet 2004 au Théâtre du Peuple de Bussang.

33. Lagarce, Jean-Luc, J'étais dans ma maison et j'attendais que la pluie vienne, Paris : Théâtre Ouvert, tapuscrit 81, 1995., p. 65.

34. Borie, Monique, Le Fantôme ou le théâtre qui doute, Arles, Actes Sud / Académie expérimentale des théâtres, coll. « Le temps du théâtre » (dir. Georges Banu), 1997, pp. 34-35.

35. Blanchot, Maurice, L'Espace littéraire, Paris, Gallimard, coll. Folio Essais, 1955, p. 344-345.

36. Ibid., p. 348.

37. Ibid., p. 348.

38. «Ce que nous avons appelé les deux versions de l'imaginaire, ce fait que l'image peut certes nous aider à ressaisir idéalement la chose, qu'elle est alors sa négation vivifiante, mais que, au niveau où nous entraîne la pesanteur qui lui est propre, elle risque aussi constamment de nous renvoyer, non plus à la chose absente, mais à l'absence comme présence, au double neutre de l'objet en qui l'appartenance au monde s'est dissipée. » (Blanchot, Maurice, ibid., p. 353)

39. Abirached, Robert, op. cit., p. 9.

40. Sarrazac, Jean-Pierre, L'Avenir du drame, Belfort, Circé, coll. Poche, 1999, p. 87.

41. Ryngaert, Jean-Pierre, op. cit., pp. 105-106.

42. L'expression est de Marie-Isabelle Boula de Mareuil: voir l'article «Rejouer "l'histoire d'avant ", étude des Serviteurs et d'Histoire d'amour (derniers chapitres)», in Problématiques d'une oeuvre, op. cit., (p. 113-131), p. 113.

43. Sermon, Julie, L'effet-figure, op. cit., p. 14.

44. Ibid., p. 14.

45. L'expression est de Julie Sermon in L'effet-figure, op. cit., pp. 174-175.

46. Sermon, Julie, op. cit., p. 44.

47. Lagarce, Jean-Luc, Le Pays lointain, op. cit., p. 313.

48. Ibid., p. 313.

49. Ibid., p. 314.

50. Ibid., p. 293-294.

51. Ibid., p. 282-283.

52. Ibid., p. 304-305.

53. Ibid., p. 315.

54. Sermon, Julie, «L'entre-deux lagarcien » in Problématiques d'une oeuvre, op.cit., p. 74.

55. Lagarce, Jean-Luc, Le Pays lointain, op.cit., p. 316.

56. Ibid., p. 317.

57. Ibid., p. 318.

58. Voir Thibaudat, Jean-Pierre, op.cit., p. 277.

59. Le théâtre de J-L. Lagarce est traversé de références à l'écriture. Cette dimension poïetique du texte est peu présente dans les trois oeuvres étudiées ici. Dans d'autres pièces, et notamment dans Histoire d'amour (derniers chapitres) ou La Photographie le hors-scène peut être analysé comme un « espace de l'écrit » dans la mesure où les références au travail d'écriture et aux changements d'état du texte dramatique sont fréquentes. La présence de l'écriture est plus ténue notre corpus, elle est liée à la figure de Louis, qui « sait écrire ", selon les membres de sa famille réelle. Dans Le Pays lointain on peut repérer une référence plus précise à cette pratique. Un Garçon, tous les garçons fait remarquer à Louis : «Je ne sais plus avec exactitude, on note mal, tu notes mal, tu as écrit ça, « un gigolo efficace » (Lagarce, Jean-Luc, Théâtre complet IV, op.cit., p. 316).

60. Sermon, Julie, « L'entre-deux lagarcien », in Problématiques d'une oeuvre, op. cit., p. 74.

61. Id., p. 74.

62. Ducrot, Oswald, Le dire et le dit, Paris, Ed. de Minuit, 1984.

63. Sermon, Julie, op. cit., p. 17. 
64. «Le drame est absolu. Pour qu'il puisse être un pur système de relations, c'est-à-dire pour être dramatique, il faut avant tout qu'il soit détaché de tout ce qui lui est extérieur. Il ne connaît rien d'autre que lui-même. » (Szondi, Peter, Théorie du drame moderne, Belfort, Circé, coll. Penser le théâtre (dir. J-P. Sarrazac), (1ère éd. Frankfurt und Main, Suhrkamp Verlag, 1965), 2006, p. 14.) 65. Ryngaert, J-P, Lire le théâtre contemporain, op. cit., p. 176.

66. Dubor, Françoise, article « Temps mort ", in Problématiques d'une oeuvre, op. cit., (p. 157-176), p. 173.

67. Mondzain, M-J., Images, icône, économie, citée par J. Sermon in L'effet-figure : états troublés du personnage contemporain, op. cit., p. 43.

68. Pavis, Patrice, Le théâtre contemporain, Analyse des textes, de Sarraute à Vinaver, op.cit., pp. 187-188.

69. Ryngaert, Jean-Pierre, op. cit., p. 102-103.

70. Lagarce, Jean-Luc, «Vivre le théâtre et sa vie » (Mégaphonie, deuxième - 4, France Culture, émission de L. Attoun, $\mathrm{n}^{\circ} 125$, septembre 1995), Théâtre / Public n 129, mai-juin 1996, p. 7, cité par Geneviève Jolly in Problématiques d'une oeuvre, op. cit., p. 19.

71. Lehmann, Hans-Thies, Le Théâtre posdramatique, Paris, L'Arche, 2002, p. 35.

72. Ibid., p. 35.

73. Ibid., p. 36 .

74. J. Sermon précise qu'elle emploie ce terme en référence à D.W. Winnicott, Jeu et réalité. L'espace potentiel.

75. Sermon, Julie, op. cit., p. 320.

76. Pavis, Patrice, Dictionnaire du théâtre, op. cit., article « hors-scène », p. 163.

77. Id., p. 163.

78. Lehmann, H-T., op. cit., p. 15.

INDEX

Mots-clés : hors-scène, sujet, Lagarce (Jean-Luc) 